\title{
Measuring starspot temperature from line-depth ratios ${ }^{\star}, \star \star$
}

\section{Simultaneous modeling of light and temperature curves}

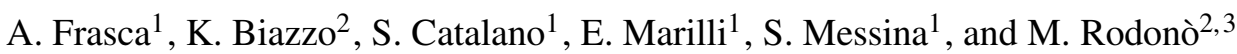 \\ 1 INAF - Catania Astrophysical Observatory, via S. Sofia 78, 95123 Catania, Italy \\ e-mail: afr@ct.astro.it \\ 2 Dep. of Physics and Astronomy, University of Catania, via S. Sofia 78, 95123 Catania, Italy \\ 3 INAF Headquarters, viale del Parco Mellini 84, 00136 Rome, Italy
}

Received 31 May 2004 / Accepted 31 October 2004

\begin{abstract}
We present and apply to VY Ari, IM Peg and HK Lac a new method to determine spot temperatures $\left(T_{\mathrm{sp}}\right)$ and areas $\left(A_{\text {rel }}\right)$ from the analysis of simultaneous light curves and temperature modulations deduced from line-depth ratios. A spot model, developed by us, has been applied to light and temperature curves. Grids of solutions with comparable $\chi$-square have been found for a wide range of spot temperatures. The behavior of the solution grids for temperature and light curves in the $T_{\mathrm{sp}}-A_{\text {rel }}$ plane is very different and a rather small and unique intersection area can be found. In our spot-model we used spectral energy distributions (SEDs) based on the Planck law and on model atmospheres to evaluate the flux ratio between spots and unspotted photosphere and we found higher spot temperatures with SEDs based on model atmospheres than on the Planck law.
\end{abstract}

Key words. stars: activity - stars: late-type - stars: individual: VY Ari, HK Lac, IM Peg - techniques: spectroscopic

\section{Introduction}

The photometric variability of RS CVn and BY Dra binaries, and of young low-mass main-sequence stars is currently interpreted in terms of dark spots on their photospheres.

Dark, cool stellar spots, like solar spots, are supposed to be the result of the blocking effect on convection caused by magnetic flux-tube emersion. Starspot temperature therefore represents an important parameter for the investigation of stellar magnetic activity and for comparison with the solar case. A precise determination of the starspot temperature, relying only on light curve inversion techniques, is strongly hampered by the lack of solution uniqueness. Although the combination of light curves with their color index variation is helpful to constrain the model parameters, the method is not very efficient (e.g., Vogt 1981; Poe \& Eaton 1985; Eaton \& Poe 1986), and more sensitive diagnostics are required.

Following a suggestion of Ramsey \& Nations (1980), Neff et al. (1995) and O'Neal et al. (1996) have used the TiO bands at 7055 and $8860 \AA$ to measure the effective temperatures and area coverages of starspots on slowly rotating active stars. They were able to derive spot temperatures with an estimated precision of $100-200 \mathrm{~K}$ for some active stars. For the very

\footnotetext{
^ Based on observations collected at Catania Astrophysical Observatory, Italy.

$\star \star$ Appendix A is only available in electronic form at http://www.edpsciences.org
}

active RS CVn-type star II Peg they found a spot temperature of about $3500 \mathrm{~K}$ and a total spot coverage between $43 \%$ and $55 \%$. Such large spot filling factors were also found for other RS CVn-type stars. However, the TiO band intensity becomes very weakly temperature dependent at $T \geq 3800 \mathrm{~K}$, so that this technique is sensitive only to very cool starspots. In addition, as pointed out by O'Neal et al. (1998), TiO bands are not strong enough to be clearly detected at $T>4000 \mathrm{~K}$, which can be considered the upper limiting temperature for the method applicability. Berdyugina (2002) stressed the usefulness of molecular bands to obtain good estimates of the spot coverage on stellar surfaces, provided that a proper synthesis of the bands is done, taking into account true element abundances, blends and Doppler shifts. In particular, she found that the spot distribution on IM Peg with a total spot coverage of about $13 \%$, as recovered from Doppler Imaging, was able to reproduce the intensity and shape of $\mathrm{TiO}$ molecular bands in any given spectrum and at any rotational phase.

In a previous paper (Catalano et al. 2002a; hereafter Paper I), we demonstrated that line depth ratios (LDRs) are powerful tools for detecting temperature variations in active RS CVn binaries. We presented the results of temperature modulation of three active stars and proposed a simple method for determining the lower limits of spot filling-factors and for estimating spot temperatures. Furthermore, we have shown that the temperature and spot coverage can be simply constrained by a combined analysis of the temperature and photometric 
variation amplitudes (Catalano et al. 2002b). Although the method leads to a unique solution, it is not able to take into account projection effects due to the latitudinal location of spots and it is strongly biased by the assumption on the value of the unspotted star magnitude.

In the present paper we will show that it is possible to solve for spot temperature and size by means of a spot-model simultaneously applied to the temperature measurements obtained from LDRs and simultaneous light curves. The method is applied to three RS CVn systems whose temperature variations have already been analyzed in Paper I, namely VY Ari, IM Peg and HK Lac.

In Sect. 2 the observations are briefly described, and the data analysis is summarized in Sect. 3. Section 4 deals with the spot modeling and the discussion of the results is presented in Sects. 5 and 6.

\section{Targets selection and observations}

We selected non-eclipsing single-lined binary systems for this study, namely VY Ari, IM Peg and HK Lac. The choice of such systems was suggested by the conflicting requirements of high activity degree and low rotation rate. A high activity level is a key requirement for detecting a significant light and temperature modulation. VY Ari, IM Peg and HK Lac satisfy this requirement, since their light modulations have rather large amplitudes $(0 \cdot 2-0 \cdot 4)$. A low rotation rate is a key requirement for a reliable line depth measurement, that becomes a very difficult or impossible task when lines are too broad, causing strong profile blending. In addition, a low $v \sin i$ will not produce any remarkable Doppler-shifting bump along the spectral line profiles, which would affect the LDRs.

In Table 1 we list the main parameters of the active stars studied in this paper (some of these values have been obtained in Paper I). Since the rotation period of some active stars is slightly variable from season to season, due to the latitude drift of the active regions and differential rotation, we adopted the period obtained by applying a Fourier periodic analysis to the photometric data used in the present work. This period is usually called "photometric period" $\left(P_{\text {phot }}\right)$ and is representative of the star average rotation rate at the spots latitudes. Other relevant parameters in our analysis are $T_{\max }, V_{\max }$ and $V_{\mathrm{ph}} . T_{\max }$ is the maximum temperature we derive for the star (see Paper I) and can be considered as the true photospheric temperature if we neglect the possible contribution of spots evenly distributed over the photosphere or polar caps, i.e. those features that do not produce any light modulation. $V_{\max }$ is the minimum visual magnitude (maximum brightness) recorded in the literature and $V_{\mathrm{ph}}$ is the visual magnitude at maximum in our light curves.

The spectroscopic observations and their reduction have been described in Paper I.

\subsection{Photometry}

Simultaneous photometric data were obtained with the 80-cm Automated Photometric Telescope (APT) of Catania Observatory at the M. G. Fracastoro station (Serra La Nave,
Table 1. Main parameters of observed active stars.

\begin{tabular}{lccc}
\hline \hline Parameter & HK Lac & IM Peg & VY Ari \\
\hline HD & 209813 & 216489 & 17433 \\
Sp. type & K0III & K2III-II & K3-4V-IV \\
$v \sin i\left(\mathrm{~km} \mathrm{~s}^{-1}\right)$ & $23.0^{a}$ & $25.6^{b}$ & $8.6^{c}$ \\
$P_{\text {rot }}($ days $)$ & $24.4284^{d}$ & $24.4936^{e}$ & $16.1996^{e}$ \\
$T_{\max }(\mathrm{K})$ & $4765^{f}$ & $4666^{f}$ & $4916^{f}$ \\
$V_{\max }(\mathrm{mag})$ & $6.66^{h}$ & $5.55^{i}$ & $6.69^{l}$ \\
$V_{\text {ph }}(\mathrm{mag})$ & $6.73^{f}$ & $5.72^{f}$ & $6.84^{f}$ \\
\hline \multicolumn{4}{c}{ Ephemerids } \\
\hline HJD0 & $2440017.17^{g}$ & $2443734.0^{e}$ & $2451856.0^{f}$ \\
$P_{\text {phot }}$ (days) & $24.283^{f}$ & $24.789^{f}$ & $16.292^{f}$ \\
\hline
\end{tabular}

${ }^{a}$ Randich et al. (1994).

${ }^{b}$ De Medeiros \& Mayor (1999).

${ }^{c}$ Bopp et al. (1989).

${ }^{d}$ Orbital period (Gorza \& Heard 1971).

${ }^{e}$ Strassmeier et al. (1997).

${ }^{f}$ Present work.

${ }^{g}$ Strassmeier et al. (CABS 1993).

${ }^{h}$ Oláh et al. (1997).

${ }^{i}$ Berdyugina et al. (1999).

${ }^{l}$ Strassmeier \& Bopp (1992).

Mt. Etna). It is an $\mathrm{f} / 8$ Cassegrain telescope equipped with a Hamamatzu R1414 SbCs photo-multiplier and Johnson's standard $U B V$ filters. The observations, corrected for the atmospheric extinction, are given in the Johnson standard system. The typical precision of $V$-band photometric measurements is about 0.01 . References to the observing procedure and data reduction may be found in Rodonò et al. (2000) and Cutispoto et al. (2001).

\section{Data analysis}

For temperature determinations we selected 15 unblended spectral lines (listed in Table 3 of Paper I) forming 10 pairs suitable for LDRs in the spectral region around $6200 \AA$. Line-depth ratios at different rotational phases were obtained for each active star (VY Ari, IM Peg, HK Lac) and then they were converted into temperatures using appropriate calibrations based on reference stars (Paper I). We derived a temperature curve for each active star by means of the weighted average of all temperature curves obtained from individual LDRs. As shown in Appendix A, at each rotational phase the average temperature is related to the photospheric temperature of the star, to the spot temperature and filling factor, and to the flux ratio between spot and photosphere at the observation wavelength. This allows us to apply a spot model to the average temperature curve instead of modelling each individual LDR curve.

The standard error of the weighted mean has been computed on the basis of the errors $\sigma_{T_{\mathrm{i}}}(\phi)$ in each LDR-derived $T_{\mathrm{i}}(\phi)$ according the usual formula (see, e.g., Topping 1972).

The average effective temperature and simultaneous photometry of VY Ari is plotted in Fig. 1 as a function of the rotational phase, determined from the initial epoch, HJD0, and 

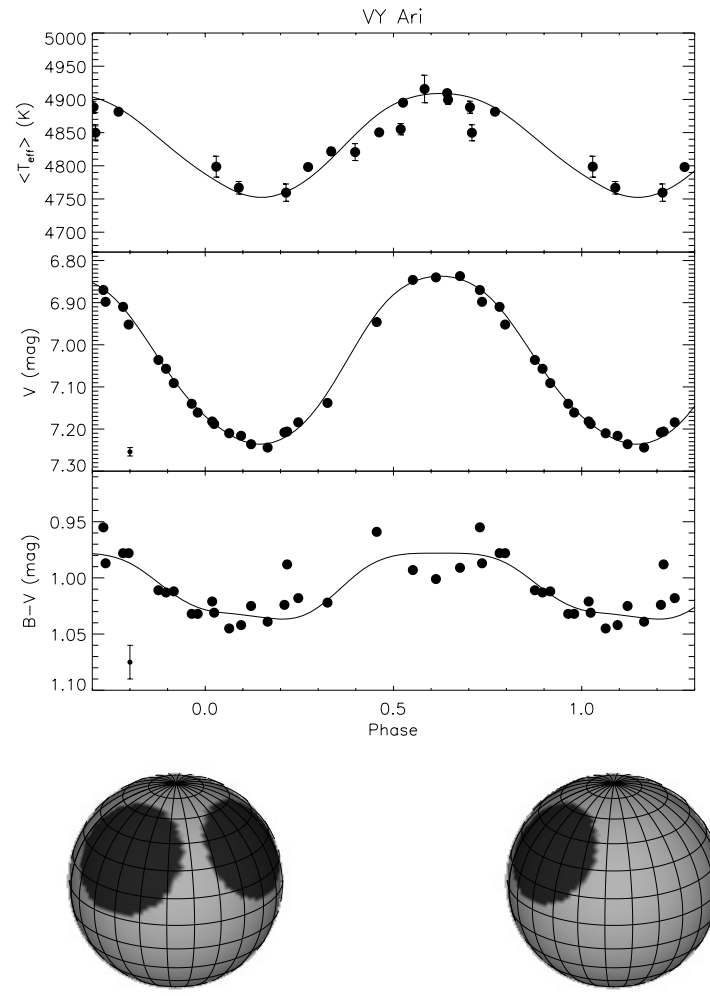

$\phi=0.15$

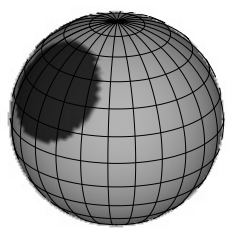

$\phi=0.85$

Fig. 1. Observed (dots) and synthetic (full lines) light and temperature curves of VY Ari. The $B-V$ curve is also shown in the lower box with the solution superimposed for comparison. A schematic map of the starspot distribution, as seen at two different rotational phases, is also shown.

the rotational period, $P_{\text {phot }}$, listed in Table 1. The temperature varies between $4760 \mathrm{~K}$ and $4916 \mathrm{~K}$, with a $\Delta\left\langle T_{\text {eff }}\right\rangle \simeq 160 \mathrm{~K}$. The $V$ light curve has a slightly asymmetric shape with a flat top $\left(V_{\mathrm{ph}}=6.84\right)$ and a variation amplitude of 0.41 , which is comparable to the maximum amplitude found by Strassmeier \& Bopp (1992). The temperature rotational modulation closely follows in phase the $V$ light curve, i.e. the temperature maximum and the brightness maximum occur both around $\phi \simeq 0^{\mathrm{p}} .6$, while the temperature and brightness minima both occur at $\phi \simeq$ $0^{\mathrm{p}} .15$. The strong correlation between temperature and brightness confirms the hypothesis of cool photospheric spots as the primary cause of the observed variations.

A similar correlation between magnitude and the $\lambda 6421.36$ Fe I- $\lambda 6419.95$ Fe I depth-ratio had been already noticed by Strassmeier (1994) in HD 106225.

The average temperature of IM Peg (Fig. 2) displays a maximum $T_{\max }=4666 \mathrm{~K}$ at $\phi \simeq 0^{\mathrm{p} .5}$ and a variation amplitude $\Delta\left\langle T_{\text {eff }}\right\rangle \simeq 120 \mathrm{~K}$. The light curve exhibits a very similar shape, with a slow rising phase from the light minimum $\left(\phi \simeq 0^{\mathrm{p}} .95\right)$ to the maximum $(\phi \simeq 0.5)$ and a faster decay. The changing slope in the descending branch of the light curve, at about phase 0.75 , is indicative of a complex spot configuration, with more than two active longitudes. The amplitude of the light curve is $\Delta V \simeq$ 0 . 20 , which is typical for this active star, but amplitude values up to $\simeq 0$. 4 have been observed (Strassmeier et al. 1999; Oláh et al. 2000).

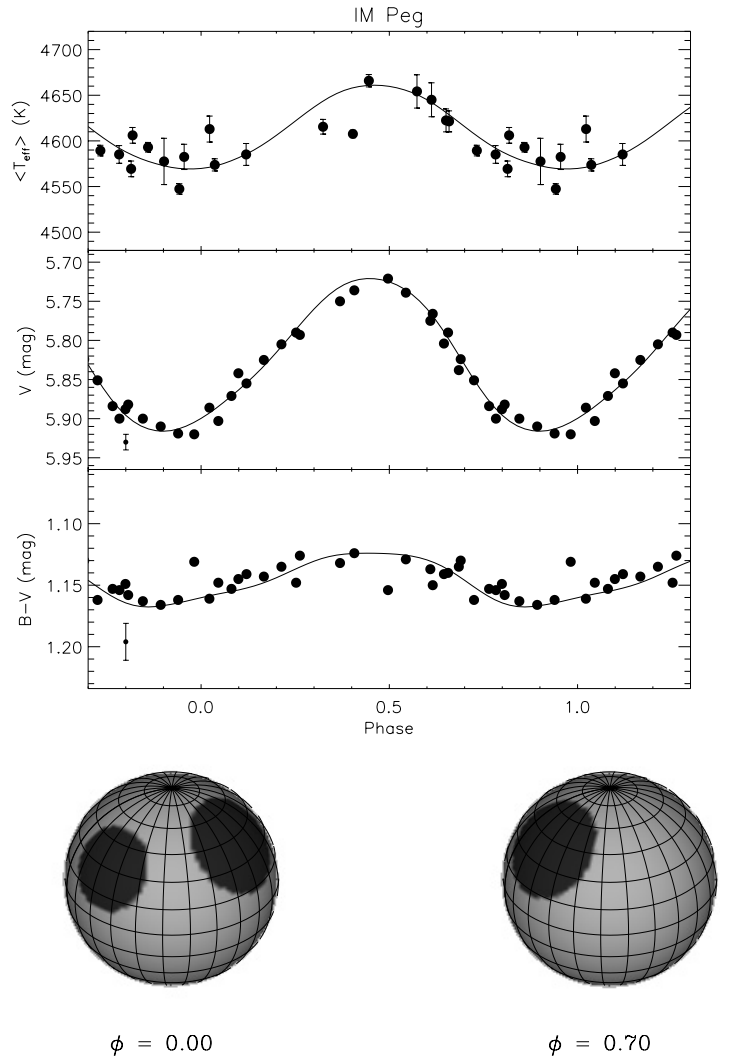

Fig. 2. Observed (dots) and synthetic (full lines) light and temperature curves of IM Peg. The $B-V$ curve is also shown in the lower box with the solution superimposed for comparison. A schematic map of the starspot distribution, as seen at two different rotational phases, is also shown.

For HK Lac the mean-temperature curve and the light curve are also in phase to a very high degree (Fig. 3). Both curves display very similar shapes with a fast rising phase from the minimum $\left(\phi \simeq 0^{\mathrm{p}} .0\right)$ to the maximum $\left(\phi \simeq 0^{\mathrm{p}} .4\right)$ and a slower decay. The amplitude of the temperature curve is $\Delta\left\langle T_{\text {eff }}\right\rangle \simeq 130 \mathrm{~K}$, with a maximum value of $4765 \mathrm{~K}$. The light curve shows a variation amplitude $\Delta V=0.37$, which is greater than the highest amplitude observed by Oláh et al. (1997) in their light curves spanning 30 years from 1967.

\section{The spot model}

The availability of simultaneous information on luminosity and average temperature allows us to reconstruct the starspot distribution and to determine two basic spot parameters, i.e. temperature and dimension.

In order to search for a unique solution of temperature and luminosity variations in these systems, we have used synthetic light and temperature curves, produced by an appropriate spot model developed by us in the IDL ${ }^{1}$ software environment. The model assumes circular dark spots on the surface of a spherical limb-darkened star. The latter assumption is well satisfied by the active components of the long-period systems studied here, because the gravitational distortion of the active star surface

\footnotetext{
1 Interactive Data Language.
} 

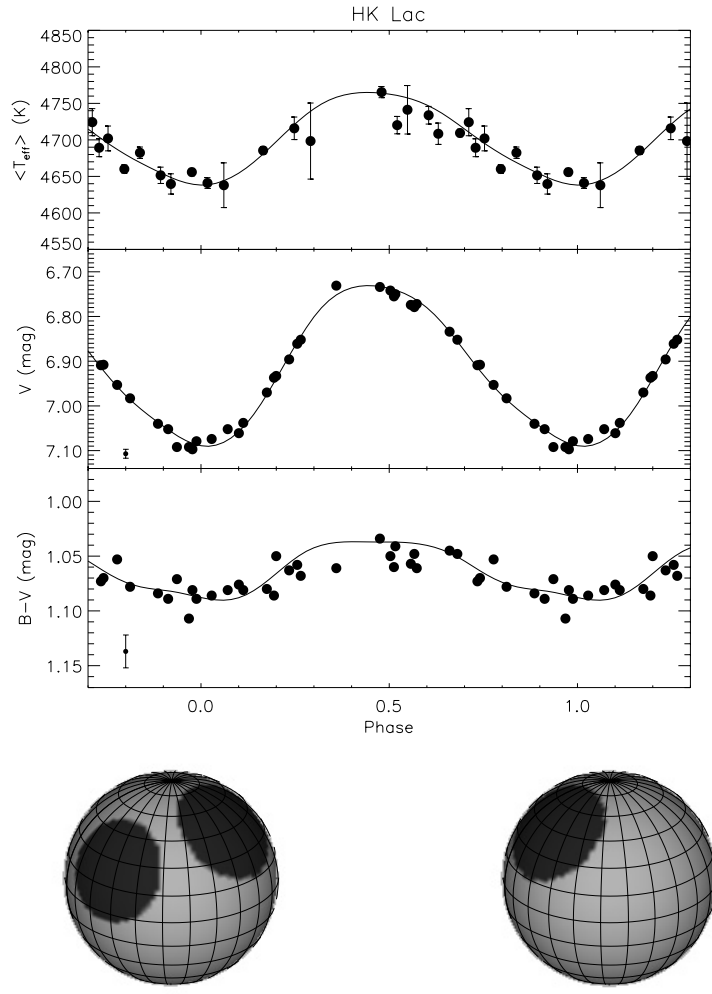

$\phi=0.00$

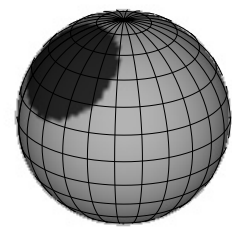

$\phi=0.70$

Fig. 3. Observed (dots) and synthetic (full lines) temperature and light curves of HK Lac. The $B-V$ curve is also shown in the lower box with the solution superimposed for comparison. A schematic map of the starspot distribution, as seen at two different rotational phases, is also shown.

induced by the unseen companion is negligible. Moreover, spots are allowed only on the primary component, the only one visible at the observing wavelengths.

Since all the observed curves are more or less asymmetric, in order to achieve a satisfactory fit at least two active regions are needed. To minimize the degrees of freedom of the model we avoided increasing the spot number. After several tests, we verified that two spots with the same temperature are sufficient for a good light-curve fitting.

The best-fit solutions of the observed light and temperature curves were searched for under the following assumptions:

- Spherical limb-darkened stars, with limb-darkening approximated by a linear relation of the type $I(\theta)=$ $I(0)(1-\mu+\mu \cos \theta)$. Values of the linear limb-darkening coefficient $\mu$ for the Johnson $V$ band were taken from Díaz-Cordovés et al. (1995), while for the continuum at $6250 \AA$ they were interpolated from the tables of Al-Naimiy (1978). Indeed, these values are always in very good agreement with the ones given by Claret et al. (1995) for the Johnson $R$ band.

- The flux ratio between spot areas and quiet photosphere, $F_{\mathrm{sp}} / F_{\mathrm{ph}}$, was computed in three different cases by using:

a) the Planck energy distribution law $B(\lambda, T)$, evaluated at the wavelengths of our spectroscopic observations for the temperature curves, and integrating $B\left(\lambda, T_{\mathrm{sp}}\right)$ and $B\left(\lambda, T_{\mathrm{ph}}\right)$ over the $V$ pass-band for the light curves;

b) the low-resolution ATLAS9 synthetic spectra (Kurucz 1993 ) for both continuum flux ratio at $\sim 6200 \AA$ and $V$ band light ratio;

c) the low-resolution PHOENIX NextGen synthetic spectra for giant stars (Hauschildt et al. 1999) for both flux ratios.

To test the reliability of our model we compared the results obtained with our code to those of Binary Maker 2.0 (Bradstreet 1993), a computer code producing synthetic light curves of binary stars, that allows us to put circular spots upon one or both components. The main limitation of Binary Maker is that the contribution of each component to the total luminosity and the flux ratio between spots and photosphere, $F_{\mathrm{sp}} / F_{\mathrm{ph}}$, is calculated by assuming black-body flux distributions. Although Binary Maker does not allow one to find the solution on the basis of a best-fit minimization, the synthetic light curves generated are in very good agreement with those obtained from our code, if the same starspot parameters are used.

As shown in Appendix $\mathrm{A}$, the flux ratio $F_{\mathrm{sp}} / F_{\mathrm{ph}}$ is a key parameter for evaluating the hemisphere-averaged temperature. We made a realistic evaluation of $F_{\mathrm{sp}} / F_{\mathrm{ph}}$ by using low-resolution synthetic spectra from ATLAS9 and PHOENIX NextGen atmosphere models, but we also used, for comparison, the black-body radiation law.

We evaluated the continuum flux at $6190 \AA$, a region close to the lines we used for LDR analysis and very weakly affected by absorption lines. The average flux in a $10 \AA$ window centered at $6190 \AA$ has been calculated for a grid of low-resolution ( $2 \AA$ ) NextGen spectra of giant stars with solar abundances (Hauschildt et al. 1999). These spectra span a $T_{\text {eff }}$ range from 2000 to $6800 \mathrm{~K}$ and a $\log g$ range from 0.0 to 3.5.

Fluxes from Kurucz low-resolution spectra, sampled at $20 \AA$, for models with solar abundance and micro-turbulence $\xi=2 \mathrm{~km} \mathrm{~s}^{-1}$ (Kurucz 1993) in the temperature range $T_{\text {eff }}=$ $3500-8750 \mathrm{~K}$ and surface gravity between 0.0 and 5.0 in steps of 0.5 , were also considered.

The continuum flux at $\lambda 6190$ for Kurucz spectra, NextGen spectra and for black-body radiation is plotted in Fig. 4 as a function of temperature. Note the flux decrement of model spectra with respect to a black-body at temperatures lower than about $4000 \mathrm{~K}$. The effect is more evident for NextGen spectra. This is a crucial point for spot modeling. The photospheres of active stars are indeed usually hotter than $4000 \mathrm{~K}$, while their spots, following the solar analogy and previous stellar studies, are presumably cooler than $4000 \mathrm{~K}$. So, the estimated flux ratio $F_{\mathrm{sp}} / F_{\mathrm{ph}}$ is dependent on the adopted flux distribution.

We also made calculations for the synthetic flux in the Johnson $V$ band by integrating the Planck radiation function and the synthetic Kurucz and NextGen spectra, weighted with the transmission curve of the $V$ filter. The result is shown in Fig. 5. The flux decrement at low temperature appears also in the $V$ flux.

To model the observed light curves we proceeded through the following steps. The spot temperature was initially fixed at the value, $T_{\mathrm{sp}} / T_{\mathrm{ph}}=0.80$. Then the two-spot solution that 


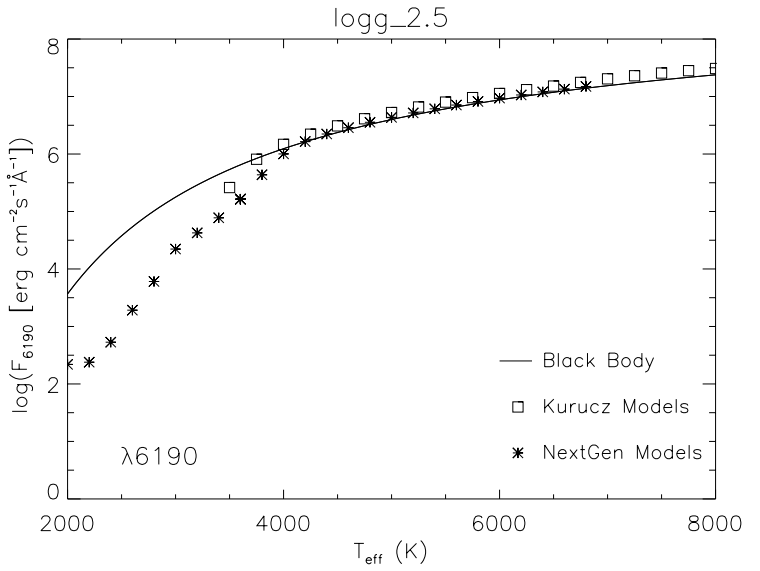

Fig. 4. Continuum flux at $\lambda 6190 \AA$ for Kurucz spectra (squares), NextGen spectra (asterisks) with $\log g=2.5$ and for black-body radiation (full line) as a function of $T_{\text {eff }}$.

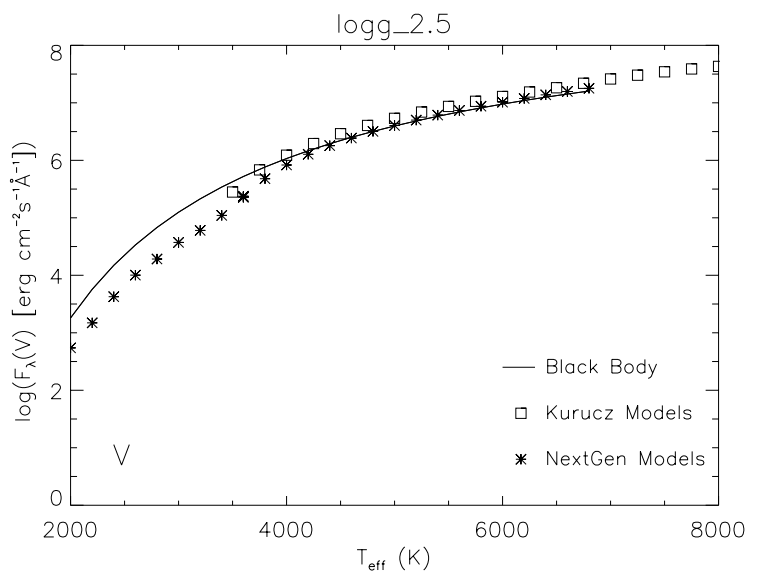

Fig. 5. $V$-band flux for Kurucz spectra (squares), NextGen spectra (asterisks) with $\log g=2.5$ and for black-body radiation (full line) as a function of $T_{\text {eff }}$.

minimizes the $\chi$-square was found. As a second step, we fixed the spot latitudes and longitudes to the values previously determined and we found the spot radii that give the best light-curve fitting for a grid of $T_{\mathrm{sp}} / T_{\mathrm{ph}}$ values. For each spot temperature, the solution that minimizes the residuals was selected. The same procedure was applied to the $T_{\text {eff }}$ curves by fixing the spot positions to the values we found from the light-curve solutions. For both diagnostics the code was run with the three options for the flux ratio (Planck, Kurucz and NextGen).

For a wide range of $T_{\mathrm{sp}} / T_{\mathrm{ph}}$ we can obtain fits of an observed light curve with comparable quality (see Fig. 6), provided that the spot area is changed, and this can largely compensate for the spot temperature changes. This is the result of the "non-uniqueness" of the spot solutions in the temperature-area domain and it also holds for the fit of the temperature curves. However, for each star and for each flux distribution, we have two different grids of solutions, one for the light curve and one for the temperature curve. As we will see in the next section, there is a unique intersection between the light and temperature grids which is almost orthogonal for Kurucz and NextGen models. It is important to note that the

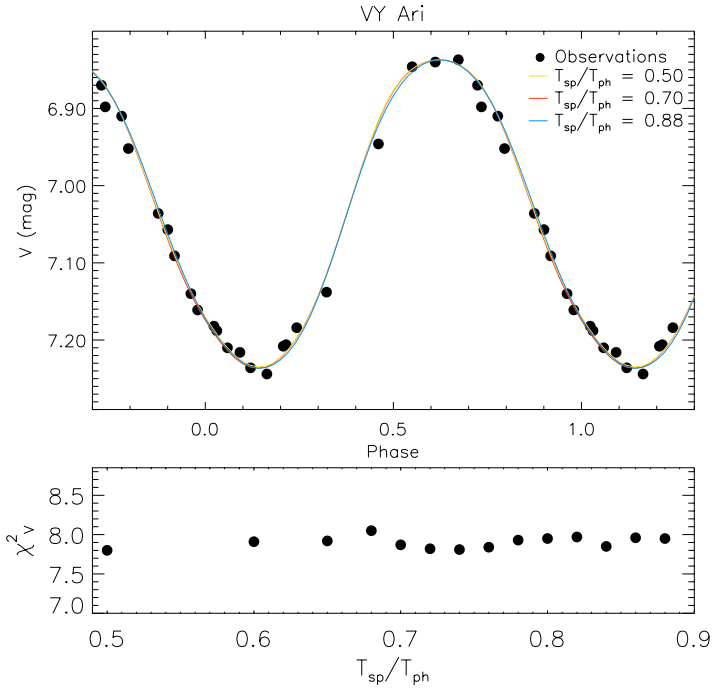

Fig. 6. Examples of synthetic curves for three spot temperatures (lines) superimposed on the $V$ light curve of VY Ari (dots). The three curves are practically indistinguishable, notwithstanding the large differences in spots temperature that are compensated by spot sizes. In the lower panel is shown the $\chi$-square as a function of the relative spot temperature $T_{\mathrm{sp}} / T_{\mathrm{ph}}$.

reliability of the solution, i.e. the $\chi^{2}$ value, remains constant for a large range of spot temperature, thus ensuring that the intersection of the solutions occurs for good quality fits (see Fig. 6).

\section{Results}

The application of our spot model code requires the knowledge of geometric and physical parameters of the active components, such as their radii, the inclination of the rotation axis with respect to the line of sight and their effective temperatures.

The inclination of the rotation axis was estimated through the $v \sin i$, the rotation period and the stellar radius. The latter was evaluated from the Hipparcos distance and the angular diameter, given by the Barnes \& Evans (1976) relation $\log \phi^{\prime \prime}=0.5134-0.2 V_{0}+0.666(B-V)_{0}$.

For VY Ari, with $V_{\max }=6.69$ (Strassmeier \& Bopp 1992), $(B-V)=0.955$ from our data at maximum brightness and an extinction $A_{\mathrm{V}}=0$ m.04 derived from a standard reddening law (Henry et al. 2000), we found an angular diameter $\phi^{\prime \prime}=$ 0.649 mas. With the Hipparcos parallax $\pi=22.73 \pm 0.89$ mas we obtained a radius $R_{*}=3.06 \pm 0.64 R_{\odot}$. From $v \sin i=8.6 \pm$ $0.7 \mathrm{~km} \mathrm{~s}^{-1}$ (Bopp et al. 1989) and $P_{\text {phot }}=16.292$ days, we deduced an inclination of the rotation axis $i=65_{-10}^{\circ+15}$.

For IM Peg, with $V_{\max }=5 \mathrm{~m} .55$ (Berdyugina et al. 1999), $(B-V)_{0}=1 \mathrm{~m}$. 101 , from our data at maximum brightness, $A_{\mathrm{V}}=$ 0.08 , and $\pi=10.33 \pm 0.76$ mas, we obtained $R_{*}=14.76 \pm$ $3.34 R_{\odot}$ which, through the $v \sin i$ value of $25.6 \pm 2.6 \mathrm{~km} \mathrm{~s}^{-1}$ (De Medeiros \& Mayor 1999), leads to an inclination of the rotation axis with respect to the line of sight $i=60_{-20}^{\circ+25}$. For comparison, Berdyugina et al. (1999) found $R_{*}=13.3 \pm 0.6 R_{\odot}$ and Strassmeier (2001) reported an inclination $i=55^{\circ}$, which are fully compatible with our values.

From the unspotted magnitude of HK Lac, $V_{\max }=6.66$ (Oláh et al. 1997), corrected for the interstellar extinction 
$A_{V}=0 .{ }^{\mathrm{m}} 12$ and the dereddened color $(B-V)_{0}=0.997$, and adopting the Hipparcos parallax of $6.62 \pm 0.61$ mas we derived a star radius $R_{*}=12.03 \pm 2.56 R_{\odot}$. Adopting the $v \sin i$ value of $23.0 \pm 3.0 \mathrm{~km} \mathrm{~s}^{-1}$ (Randich et al. 1994), we found an inclination of the rotation axis $i=65_{-15}^{\circ+20}$ which is in very good agreement with the value of $68^{\circ}$ found by Stawikowski \& Glebocki (1994).

Since for the three active stars there is no long-term monitoring of the photospheric temperature, we do not have the possibility to estimate the "unspotted" temperature from the historically-recorded maximum, as is frequently done for light-curve modelling. Thus, we had to assume the maximum brightness and temperature observed in our run as unspotted level values. Therefore we are taking into account only the unevenly distributed component of the spotted area, i.e. the component giving rise to the observed modulation. On the one hand we are possibly underestimating the total spotted area, on the other side we are maintaining consistency between the light curve and temperature curve analysis.

\subsection{VY Ari}

The maximum brightness of VY Ari at the epoch of our observations, $V_{\mathrm{ph}}=6.84$, is greater than the value of 6.90 reported in CABS, but it is lower than the maximum value of 6.69 found by Strassmeier \& Bopp (1992), that they assumed as the unspotted value.

We have assumed the maximum value of the temperature curve, $T_{\max }=4916 \mathrm{~K}$, as the effective temperature of the star photosphere. Eaton \& Poe (1986) and Strassmeier \& Bopp (1992) adopted $4260 \mathrm{~K}$ and $4600 \mathrm{~K}$, respectively, while Alekseev \& Kozlova (2001) found a temperature of $4750 \mathrm{~K}$ from color indices and synthetic spectra. The $T_{\text {eff }}$ values of $4260 \mathrm{~K}$ and $4600 \mathrm{~K}$, however, are poorly consistent with both the spectral classification of VY Ari and its color indices. From the dereddened color index at maximum brightness in our data, $(B-V)_{0}=0.944$, we can deduce, according to the Flower (1996) and Gray (1992) calibrations, an effective temperature of 4940-4960 K, which is in excellent agreement with the value of $T_{\max }$ from LDRs. The value by Alekseev \& Kozlova (2001) better agrees with our findings.

The results of the solution grids for the light curve and the temperature curve are displayed in Fig. 7, where the fractional area, $A_{\text {rel }}$, of the spotted region versus the fractional spot temperature $T_{\mathrm{sp}} / T_{\mathrm{ph}}$ is plotted. The three boxes show the results obtained with the different assumptions on the SED (Planck, Kurucz, and NextGen). In all cases, the light-curve solution grid has an exponential-like behavior because the warmer the spots are, the bigger they must be to produce the observed light variation. For the temperature-curve solutions, as stressed in Paper I, the dependence is more complex. If the spot is very $\operatorname{cool}\left(T_{\mathrm{sp}}\right.$ very small), its contribution to the mean temperature is negligible because $F_{\mathrm{sp}} / F_{\mathrm{ph}}$ in Eq. (A.5) decreases rapidly, so that the average temperature observed is near the photospheric one. As a consequence, a large spot area would be required to account for the observed temperature variation. If $T_{\mathrm{sp}} / T_{\mathrm{ph}}$ approaches unity, the average temperature is not appreciably

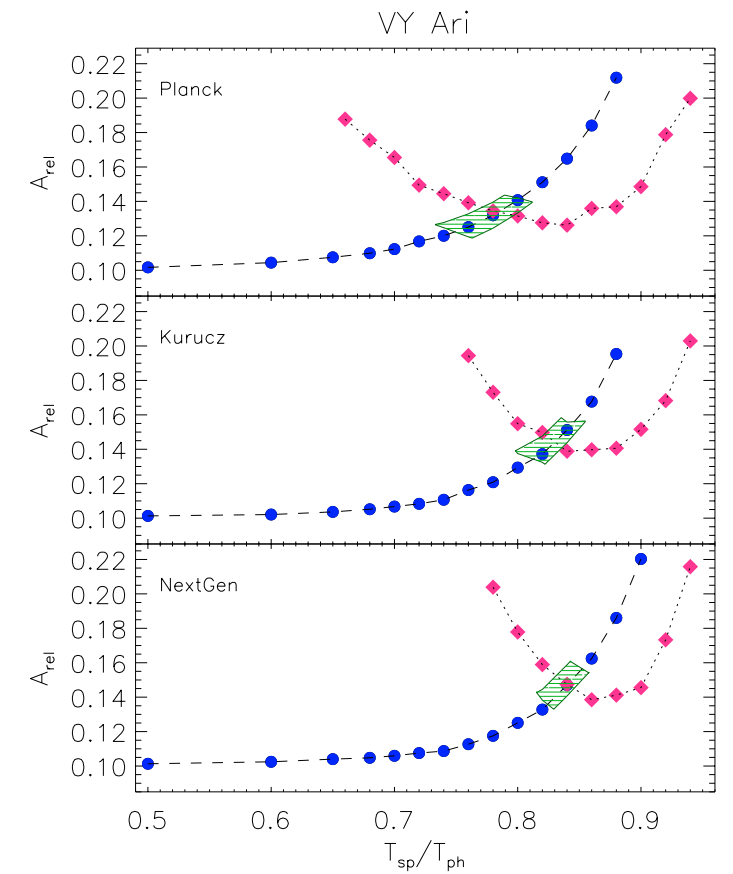

Fig. 7. Grids of solutions for VY Ari. The filled circles represent the solutions for light curve, while the filled diamonds represent the solutions for temperature curve. The hatched area, in each box, is the locus of the allowed solutions accounting for data errors.

changed by the passage of spots over the visible hemisphere and again very large spots are needed to reproduce the observed variation. This explains why the grid of solutions in the plane $T_{\mathrm{sp}} / T_{\mathrm{ph}}-A_{\text {rel }}$ passes through a minimum fractional area.

The two grids of solutions intersect for $T_{\mathrm{sp}} / T_{\mathrm{ph}}=$ 0.786, 0.830, 0.840, for Planck, Kurucz and NextGen models, respectively. At these values we can simultaneously fit both the light curve and the temperature curve.

The accuracy on $T_{\mathrm{sp}}$ and $A_{\text {rel }}$ was evaluated by adopting 0.03 and $30 \mathrm{~K}$ as average $3 \sigma$ errors for the observed light and temperature curves, respectively. The hatched area in Fig. 7 represents the locus of the allowed solutions in the plane $T_{\mathrm{sp}} / T_{\mathrm{ph}}-A_{\text {rel }}$ and leads to errors on temperature and area $\Delta \frac{T_{\mathrm{sp}}}{T_{\mathrm{ph}}}=0.016,0.024,0.018$ and $\Delta A_{\text {rel }}=0.012,0.013,0.015$ for Planck, Kurucz and NextGen models, respectively.

In Fig. 1 the observed light and temperature curves and the simultaneous solution are displayed together with a map of the spotted photosphere, as seen at two different rotational phases. In the lower box of the same figure, the observed $B-V$ color index is displayed together with the curve predicted from the simultaneous solution of $V$ and $T_{\text {eff }}$. Despite the data scatter, the agreement is rather good.

Although we will consider the results from model atmosphere solutions as the more reliable, we will discuss also the black-body solutions to better compare our results with previous works which are mainly based on the latter assumption.

For VY Ari we found $\Delta T=T_{\mathrm{ph}}-T_{\mathrm{sp}}=1052 \mathrm{~K}, 836 \mathrm{~K}$, $787 \mathrm{~K}$ for Planck, Kurucz and NextGen models, respectively (Table 2). Eaton \& Poe (1986), Strassmeier \& Bopp (1992) and Alekseev \& Kozlova (2001) had obtained 760 K, $1200 \pm 50 \mathrm{~K}$ and $1260 \div 1410 \mathrm{~K}$, respectively, from photometric data. 
Table 2. Spot configuration for VY Ari. The stellar parameters used in the solution are: unspotted effective temperature, $T_{\max }=4916 \mathrm{~K}$; limb-darkening coefficients at $V$ and at $6200 \AA, \mu_{\mathrm{V}}=0.77, \mu_{6200}=$ 0.63 ; inclination, $i=65^{\circ}$.

\begin{tabular}{lcrccc}
\hline \hline Model & Radius & Lon. $^{a}$ & Lat. & $\frac{T_{\mathrm{sp}}}{T_{\mathrm{ph}}}$ & $A_{\text {rel }}{ }^{b}$ \\
\hline Planck & 28.0 & $355^{\circ}$ & $35^{\circ}$ & $0.786 \pm 0.016$ & $0.135 \pm 0.012$ \\
" " & 31.8 & $90^{\circ}$ & $35^{\circ}$ & $0.786 \pm 0.016$ & \\
Kurucz & 29.5 & $355^{\circ}$ & $35^{\circ}$ & $0.830 \pm 0.024$ & $0.144 \pm 0.013$ \\
" " & 32.5 & $90^{\circ}$ & $35^{\circ}$ & $0.830 \pm 0.024$ & \\
NextG & 28.7 & $355^{\circ}$ & $35^{\circ}$ & $0.840 \pm 0.018$ & $0.147 \pm 0.015$ \\
" " & 34.1 & $90^{\circ}$ & $35^{\circ}$ & $0.840 \pm 0.018$ & \\
\hline
\end{tabular}

${ }^{a}$ Longitude increases with phase, and $0^{\circ}$ longitude corresponds to phase 0 ? 0 .

${ }^{b}$ Total fractional area of the two spots in units of the star surface.

Table 3. Spots configuration for IM Peg. The stellar parameters used in the solution are: unspotted effective temperature, $T_{\max }=4666 \mathrm{~K}$; limb-darkening coefficients at $V$ and at $6200 \AA, \mu_{\mathrm{V}}=0.80, \mu_{6200}=$ 0.68 ; inclination, $i=60^{\circ}$.

\begin{tabular}{lllccc}
\hline \hline Model & Radius & Lon. & Lat. & $\frac{T_{\mathrm{sp}}}{T_{\mathrm{ph}}}$ & $A_{\text {rel }}$ \\
\hline Planck & $27^{\circ} .2$ & $123^{\circ}$ & $138^{\circ}$ & $0.873 \pm 0.050$ & $0.093 \pm 0.025$ \\
" " & 21.8 & $223^{\circ}$ & $149^{\circ}$ & $0.873 \pm 0.050$ & \\
Kurucz & $28^{\circ} .5$ & $123^{\circ}$ & $138^{\circ}$ & $0.904 \pm 0.027$ & $0.106 \pm 0.020$ \\
" " & $23^{\circ} .4$ & $223^{\circ}$ & $149^{\circ}$ & $0.904 \pm 0.027$ & \\
NextG & $30^{\circ} 0$ & $123^{\circ}$ & $138^{\circ}$ & $0.915 \pm 0.030$ & $0.115 \pm 0.030$ \\
" " & $23^{\circ} .9$ & $223^{\circ}$ & $149^{\circ}$ & $0.915 \pm 0.030$ & \\
\hline
\end{tabular}

\subsection{IM Peg}

The parameters we used for the IM Peg spot model are reported in Table 3.

The effective temperature we obtained at maximum brightness is $T_{\max }=4666 \mathrm{~K}$, while Poe \& Eaton (1985), Padmakar \& Pandey (1999) and Ribárik et al. (2003) assumed $T_{\text {eff }}=$ $4440 \mathrm{~K}, 4400 \mathrm{~K}$ and $4450 \mathrm{~K}$, respectively. Indeed, from $T_{\text {eff }}-(B-V)$ calibrations and $(B-V)_{0}=1{ }^{\mathrm{m}} .101$ we find $T_{\text {eff }}=$ $4660 \mathrm{~K}$, from Flower (1996) calibration, and $T_{\text {eff }}=4650 \mathrm{~K}$ from Gray (1992), in perfect agreement with the value found from our LDR analysis.

The two groups of solutions intersect at $T_{\mathrm{sp}} / T_{\mathrm{ph}}=$ 0.873, 0.904, 0.915, for Planck, Kurucz and NextGen models, respectively (Fig. 8).

The observed temperature and light curves, together with the synthetic solution, are shown in Fig. 2. The photospheric map at two different phases is also displayed in the same figure.

For IM Peg, our method leads to $\Delta T=593 \mathrm{~K}, 448 \mathrm{~K}$, 397 K for Planck, Kurucz and NextGen models, respectively. From light-curve solutions, Poe \& Eaton (1985) obtained $\Delta T=$ $920 \pm 100 \mathrm{~K}$, while Padmakar \& Pandey (1999) get $1130 \pm$ 35 K. Ribárik et al. (2003) analyzed a large set of light and color curves of IM Peg with a spot model program using a black-body approximation. They found a rather strong indication of variation of the spot temperature along the activity cycle, with an average $\Delta T$ of about $900 \mathrm{~K}$. In particular, they

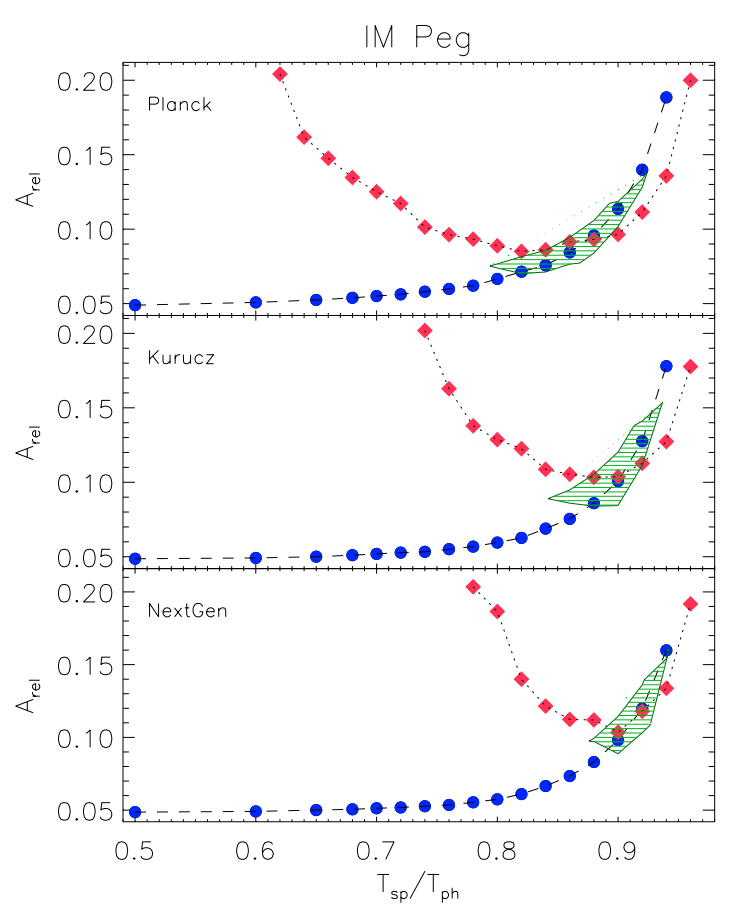

Fig. 8. Grids of solutions for IM Peg. The filled circles represent the solutions for light curve, while the filled diamonds represent the solutions for temperature curve. The hatched area, in each box, is the locus of the allowed solutions accounting for data errors.

found $\Delta T \simeq 650 \mathrm{~K}$ from observations contemporaneous to our data and this is in very good agreement with our solution in the black-body approximation.

\subsection{HK Lac}

Table 4 presents the geometric and physical parameters we used for the spot model of HK Lac, together with the spot parameters of the best solution that simultaneously fits the light curve and the temperature curve.

The effective temperature we measure at maximum brightness is $T_{\max }=4765 \mathrm{~K}$, while from $T_{\text {eff }}-(B-V)$ calibrations and $(B-V)_{0}=0.997$ we find $T_{\text {eff }}=4870 \mathrm{~K}$ from Flower (1996) calibration and $T_{\text {eff }}=4860 \mathrm{~K}$ from Gray (1992), in fairly good agreement with the value found from the LDR analysis. Vogt (1981) used a $T_{\text {eff }}=4790 \mathrm{~K}$, while Oláh et al. (1997) adopted a photospheric temperature of $4820 \mathrm{~K}$. These two values are very close to the value derived by us, unlike Poe \& Eaton's (1985) value of $4600 \mathrm{~K}$.

The two grids of solutions (for temperature and brightness) with the "Planck" SED intersect at $T_{\mathrm{sp}} / T_{\mathrm{ph}}=0.776$, i.e. $T_{\mathrm{sp}}=$ 3698 K (Fig. 9, Table 4), while from the Kurucz and NextGen spectra we derive 0.839 and 0.850 , respectively.

The $\Delta T$ values that we find for HK Lac are $1067 \mathrm{~K}, 767 \mathrm{~K}$, $715 \mathrm{~K}$ for Planck, Kurucz and NextGen models, respectively. By modeling $V$ light curves and $V-R$ color index, Vogt (1981) found $\Delta T=950 \pm 200 \mathrm{~K}$. Poe \& Eaton (1985) found instead $\Delta T \gtrsim 1080 \pm 100 \mathrm{~K}$ from $V-I_{\mathrm{C}}$ observations. Oláh et al. (1997) reports $\Delta T=1200 \pm 100 \mathrm{~K}$ from a spot model analysis in the black body approximation. 
Table 4. Spot configuration for HK Lac. The stellar parameters used in the solution are: unspotted effective temperature, $T_{\max }=4765 \mathrm{~K}$; limb-darkening coefficients at $V$ and at $6200 \AA, \mu_{\mathrm{V}}=0.80, \mu_{6200}=$ 0.68 ; inclination, $i=65^{\circ}$.

\begin{tabular}{lrrrcc}
\hline \hline Model & Radius & Lon. & Lat. & $\frac{T_{\mathrm{sp}}}{T_{\mathrm{ph}}}$ & $A_{\text {rel }}$ \\
\hline Planck & $28^{\circ} 8$ & $305^{\circ}$ & $47^{\circ}$ & $0.776 \pm 0.040$ & $0.117 \pm 0.010$ \\
" " & $27^{\circ} .2$ & $38^{\circ}$ & $25^{\circ}$ & $0.776 \pm 0.040$ & \\
Kurucz & $30^{\circ} 6$ & $305^{\circ}$ & $47^{\circ}$ & $0.839 \pm 0.025$ & $0.131 \pm 0.016$ \\
" " & 28.8 & $38^{\circ}$ & $25^{\circ}$ & $0.839 \pm 0.025$ & \\
NextG & 32.2 & $305^{\circ}$ & $47^{\circ}$ & $0.850 \pm 0.019$ & $0.138 \pm 0.016$ \\
" " & $30^{\circ} .2$ & $38^{\circ}$ & $25^{\circ}$ & $0.850 \pm 0.019$ & \\
\hline
\end{tabular}

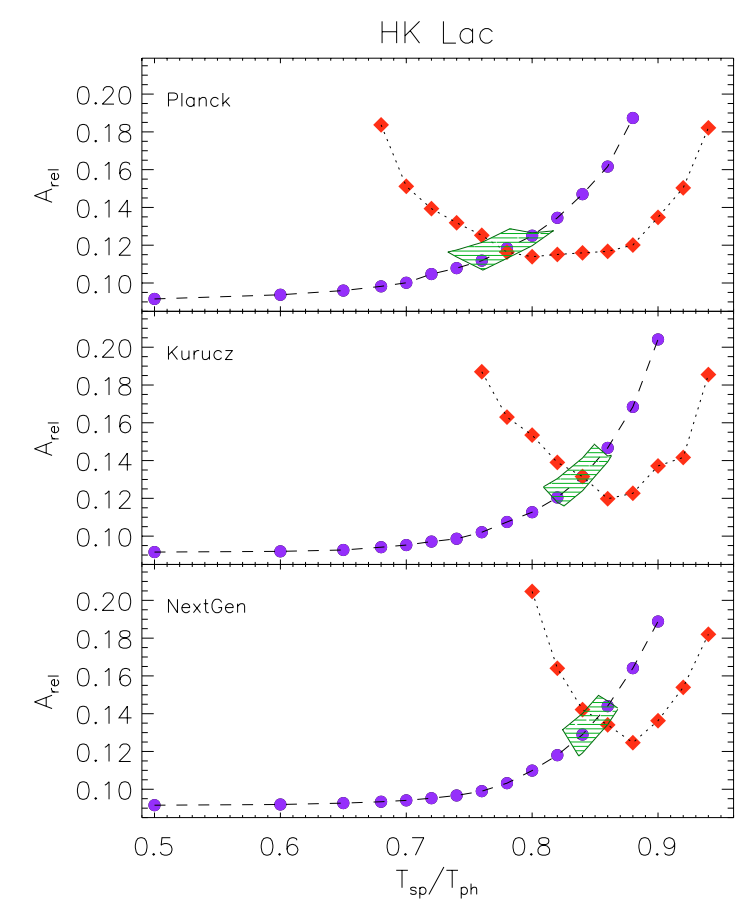

Fig. 9. Grids of solutions for HK Lac. The filled circles represent the solutions for light curve, while the filled diamonds represent the solutions for temperature curve. The hatched area, in each box, is the locus of the allowed solutions accounting for data errors.

\section{Discussion}

The grids of solutions displayed in Figs. 7-9, are characterized by two main behaviors. The solutions of the temperature curve in the $T_{\mathrm{sp}} / T_{\mathrm{ph}}-A_{\text {rel }}$ plane reach a minimum of $A_{\text {rel }}$ that, for model atmosphere SEDs, occurs at $T_{\mathrm{sp}} / T_{\mathrm{ph}}$ higher than that obtained with the black-body approximation. There is no significant difference between the grids for NextGen and Kurucz models. The width of the curve that is representative of the solution grid for temperature in the $T_{\mathrm{sp}} / T_{\mathrm{ph}}-A_{\text {rel }}$ plane decreases in going from Planck to NextGen through Kurucz SEDs. Moreover, these curves are slightly asymmetric with respect to their minimum. Also the exponential-like trend of the solution grid for the light curve changes its shape going from Planck to NextGen SED and, in particular, it becomes steeper and moves to higher values of $T_{\mathrm{sp}} / T_{\mathrm{ph}}$.
This result can be easily understood if we consider that, for a given spot temperature, the relative flux contribution of the spotted area is noticeably higher for the Planck SED than for those derived from model atmospheres (cf. Figs. 4 and 5). The lower flux ratio $F_{\mathrm{sp}}^{\mathrm{c}} / F_{\mathrm{ph}}^{\mathrm{c}}$ we deduce from Kurucz and NextGen models with respect to the black-body approximation reduces the effect of the spots on the hemisphere-averaged temperature and, consequently, requires a higher spot area for a given $T_{\mathrm{sp}}$. This behavior is more evident at low $T_{\mathrm{sp}}$ values. In the synthetic ligth curves, instead, the lower $F_{\mathrm{sp}}^{\mathrm{c}} / F_{\mathrm{ph}}^{\mathrm{c}}$ for model-atmosphere SEDs requires, for any $T_{\mathrm{sp}} / T_{\mathrm{ph}}$, a spot area smaller than in the Planck case and this effect is more evident at higher $T_{\mathrm{sp}} / T_{\mathrm{ph}}$, when the light contributed by the spotted regions is not negligible. As a consequence, the intersection between the grids of temperature-curve and light-curve solutions moves to higher values of $T_{\mathrm{sp}} / T_{\mathrm{ph}}$ in going from Planck to model-atmosphere SEDs, as is apparent in Figs. 7-9. This result explains why spot models using a black-body approximation lead to lower spot temperatures. The flux deficiency at $T<4000 \mathrm{~K}$ is the result of the inclusion of opacities of $10^{6}$ atomic lines in the model atmosphere calculations. The opacities play a very important role in any model calculation and their correct treatment is necessary to obtain reliable estimates of many physical parameters, such as effective temperature, gravity, abundances and micro-turbulence. The predictions of Kurucz and NextGen model-atmosphere spectra agree fairly well.

The spot temperatures we deduce with the Kurucz and NextGen SED are in the range 4000-4200 K, while those obtained with the Planck approximation range from about $3700 \mathrm{~K}$ to $4100 \mathrm{~K}$.

Spot temperatures derived for some active stars by means of TiO molecular bands by O'Neal et al. (1996) are in the range 3450-3850 K. In particular, for the very active RS CVn-type star II Peg, they derived $T_{\mathrm{sp}} \simeq 3500 \mathrm{~K}$ and a total spot coverage between $43 \%$ and $55 \%$, a result also found from UBVJHK photometry by Marino et al. (1999). Large spot filling factors $(20-40 \%)$ were also found for other active stars.

Solanki \& Unruh (2004) remark that, for a few active stars with nearly contemporaneous observations, the hemispheric spot coverage deduced from Doppler Imaging is sensibly lower than that deduced by O'Neal et al. (1996) from TiO bands.

A simultaneous analysis of IM Peg spectra covering the TiO bands and including absorption lines suitable for Doppler Imaging (DI) has been made by Berdyugina (2002), who paid particular attention to the treatment of element abundances, blending with $\mathrm{CN}$ lines and rotational broadening in starspot lines. She found that the spot coverage of about $13 \%$, recovered by means of DI, is able to reproduce the intensity, shape and variation of TiO bands.

The filling factors we found are consistent with values typically derived from DI maps, but the starspot temperatures are slightly higher than those derived from DI in the cores of the spotted regions (e.g., Berdyugina 2002).

A comparison of our results with the solar case show that, in these active binaries, starspots are slightly hotter than sunspot umbrae, whose temperatures typically are $\approx 3800 \mathrm{~K}$ (e.g., Priest 1982; Zirin 1988, and references therein), although they cover a much wider portion (11-15\%) of the stellar surface. 
Because the effective temperature of our targets is much lower than that of the Sun, one should consider the temperature difference $\Delta T$ or the temperature ratio $T_{\mathrm{sp}} / T_{\mathrm{ph}}$ as a more representative parameter for the comparison.

The $\Delta T$ values we found for the active stars in this study $(450-850 \mathrm{~K})$ are much smaller than the typical values of sunspot umbrae, but are significantly larger than those of sunspot penumbrae. These starspot temperatures are probably more representative of a weighted average of temperatures of umbra and penumbra, if the solar analogy holds also for starspots in very active stars.

If the starspots can be represented by two components of different temperatures and fractional areas in analogy with sunspots, our method would be more sensitive to the detection of starspot "penumbrae", due to their higher areas (a factor 3-5 with respect to umbrae; see, e.g., Solanki \& Unruh 2004, and reference therein) and the higher continuum flux, which gives more weight to the penumbrae in the observed hemisphere averaged spectrum.

\section{Conclusion}

We have considered the spottedness of three active single-lined RS CVn systems by using simultaneous light curves and temperature curves from spectroscopic line-depth ratios. We have shown that these two spot diagnostics are correlated to a very high degree, with lower temperature corresponding to lower luminosity.

The application of a spot model developed by us has permitted to determine in an univocal way the spot temperature and area.

We have searched for simultaneous solutions of the temperature and light curves by using the spectral energy distribution (SED) predicted by model atmospheres (Kurucz and NextGen) to evaluate the continuum flux ratio between the spots and the quiet photosphere. We have also computed the spot temperatures deduced under the black-body approximation for the flux ratio to compare our findings with previous results on the same stars. We found that the spot temperatures derived with the black-body SED are lower than those obtained with the model atmosphere SED. The results from Kurucz and NextGen models are instead in good agreement.

The temperature difference between spots and photosphere, a parameter tied to the blocking of the convection produced by starspot magnetic fields, is in the range $450-850 \mathrm{~K}$. These values are significantly smaller than those typical of sunspot umbrae, and are probably more representative of a weighted average of umbral and penumbral temperatures.

If we assume that the solar penumbral/umbral area and flux ratio holds also for such very active stars, our method appears to be more sensitive to the detection of warmer starspot regions similar to solar penumbrae.

Acknowledgements. The authors are grateful to the anonymous Referee for a careful reading of the paper and valuable comments. This work has been supported by the Italian Ministero dell'Istruzione, Università e Ricerca (MIUR) and by the Regione Sicilia. This research has made use of SIMBAD and VIZIER databases, operated at CDS, Strasbourg, France.

\section{References}

Al-Naimiy, H. M. 1978, Ap\&SS, 53, 181

Alekseev, I. Y., \& Kozlova, O. V. 2001, Astrophysics, 44, 429

Barnes, T. G., \& Evans, D. S. 1976, MNRAS, 174, 489

Berdyugina, S. V., Ilyin, I., \& Tuominen, I. 1999, A\&A, 347, 932

Berdyugina, S. V. 2002, AN, 323, 192

Bopp, B. W., Saar, S. H., Ambruster, C., et al. 1989, ApJ, 339, 1059

Bradstreet, D. H. 1993, Binary Maker 2.0 User Manual, Contact Software

Catalano, S., Biazzo, K., Frasca, A., \& Marilli, E. 2002a, A\&A, 394, 1009 (Paper I)

Catalano, S., Biazzo, K., Frasca, A., et al. 2002b, Astron. Nachr., 323, 260

Claret, A., Díaz-Cordovés, J., \& Gimenez, A. 1995, A\&AS, 114, 247

Cutispoto, G., Messina, S., \& Rodonò, M. 2001, A\&A, 367, 910

De Medeiros, J. R., \& Mayor, M. 1999, A\&AS, 139, 433

Díaz-Cordovés, J., Claret, A., \& Gimenez, A. 1995, A\&AS, 110, 329

Eaton, J. A., \& Poe, C. H. 1986, IBVS, 2846

Flower, P. J. 1996, ApJ, 469, 355

Gorza, W. L., \& Heard, J. F. 1971, Publ. David Dunlap Obs., 3, 107

Gray, D. F. 1992, The Observation and Analysis of Stellar Photospheres, 2nd ed. (Cambridge University Press), 344

Hauschildt, P. H., Allard, F., Ferguson, J., Baron, E., \& Alexander, D. R. 1999, ApJ, 525, 871

Henry, G. W., Fekel, F. C., Henry, S. M., \& Hall, D. S. 2000, ApJS, 130,201

Kurucz, R. L. 1993, ATLAS9 Stellar Atmosphere Programs and $2 \mathrm{~km} \mathrm{~s}^{-1}$ grid, Kurucz CD-ROM No. 13

Marino, G., Rodonò, M., Leto, G., \& Cutispoto, G. 1999, A\&A, 352, 189

Neff, J. E., O’Neal, D., \& Saar, S. H. 1995, ApJ, 452, 879

Oláh, K., Kövári, Zs., Bartus, J., et al. 1997, A\&A, 321, 811

Oláh, K., Kolláth, Z., \& Strassmeier, K. G. 2000, A\&A, 356, 643

O’Neal, D., Saar, S. H., \& Neff, J. E. 1996, ApJ, 463, 766

O’Neal, D., Neff, J. E., \& Saar, S. H. 1998, ApJ, 507, 919

Padmakar, \& Pandey, S. K. 1999, A\&AS, 138, 203

Poe, C. H., \& Eaton, J. A. 1985, ApJ, 289, 644

Priest, E. R. 1982, Solar magneto-hydrodynamics (Reidel D. Publishing Company), 48

Randich, S., Giampapa, M. S., \& Pallavicini, R. 1994, A\&A, 283, 893

Ramsey, L. W., \& Nations H. L. 1980, ApJ, 239, L121

Ribárik, G., Oláh, K., \& Strassmeier, K. G. 2003, Astron. Nachr., 324, 202

Rodonò, M., Messina, S., Lanza, A. F., Cutispoto, G., \& Teriaca, L. 2000, A\&A, 358, 624

Strassmeier, K. G. 2001, in Cool Stars, Stellar Systems, and the Sun, ed. R. J. Garciá López, R. Rebolo, \& M. R. Zapatero Osorio, ASP Conf. Ser., 223, 271

Solanki, S. K., \& Unruh, Y. C. 2004, MNRAS, 348, 307

Stawikowski, A., \& Glebocki, R. 1994, Acta Astr., 44, 393

Strassmeier, K. G., \& Bopp, B. W. 1992, A\&A, 259, 183

Strassmeier, K. G., Hall, D. S., Fekel, F. C., \& Scheck, M. (CABS) 1993, A\&AS, 100, 173

Strassmeier, K. G. 1994, A\&A, 281, 395

Strassmeier, K. G., Bartus, J., Cutispoto, G., \& Rodonò, M. 1997, A\&AS, 125, 11

Strassmeier, K. G., Serkowitsch, E., \& Granzer, T. 1999, A\&AS, 140, 29

Topping, J. 1972, Errors of Observation and Their Treatment (Chapman \& Hall Ltd.), 89

Vogt, S. S. 1981, ApJ, 150, 327

Vogt, S. S., Penrod, G. D., \& Hatzes, A. P. 1987, ApJ, 321, 496

Zirin, H. 1988, Astrophysics of the Sun (Cambridge University Press), 324 
A. Frasca et al.: Measuring starspot temperature from line-depth ratio. II., Online Material $p 1$

\section{Online Material}


A. Frasca et al.: Measuring starspot temperature from line-depth ratio. II., Online Material $p 2$

\section{Appendix A: The hemisphere-averaged temperature and luminosity}

To properly analyze the temperature variation determined from LDRs, we have to consider that we are deducing a "temperature" from a diagnostic that makes use of a "composite" spectrum which is the result of the combination of the spectrum of the star photosphere and that produced by the starspots. To make this point clear, let us consider a schematic model of a star with a photospheric temperature $T_{\mathrm{ph}}$ and a spotted region with temperature $T_{\mathrm{sp}}$ of relative area $A_{\text {rel }}$.

Let us take the spectrum of the standard star $\kappa$ Gem (G8 III, $T_{\text {eff }}=5000 \mathrm{~K}$ ), displayed in the upper part of Fig. A.1, as the photospheric spectrum without any contamination from spotted regions. The line depth ratio is defined as

$r_{\mathrm{ph}}=\frac{d_{1}^{\mathrm{ph}}}{d_{2}^{\mathrm{ph}}}=\frac{1-\frac{F_{\mathrm{ph}}\left(\lambda_{1}\right)}{F_{\mathrm{ph}}^{\mathrm{c}}}}{1-\frac{F_{\mathrm{ph}}\left(\lambda_{2}\right)}{F_{\mathrm{ph}}^{\mathrm{c}}}}=\frac{F_{\mathrm{ph}}^{\mathrm{c}}-F_{\mathrm{ph}}\left(\lambda_{1}\right)}{F_{\mathrm{ph}}^{\mathrm{c}}-F_{\mathrm{ph}}\left(\lambda_{2}\right)}$,

where $d_{1,2}^{\mathrm{ph}}$ are the line depths, $F_{\mathrm{ph}}\left(\lambda_{1}\right)$ is the residual flux at the bottom of the line 1 and $F_{\mathrm{ph}}^{\mathrm{c}}$ is the continuum flux in this spectral region (see Fig. A.1).

Similar relations hold for the spectrum of the spot that has been represented by $\beta \mathrm{Cnc}$, $\left(\mathrm{K} 4 \mathrm{III}, T_{\text {eff }}=3900 \mathrm{~K}\right)$ in the bottom plot of Fig. A.1.

For a spotted star, we observe a composite spectrum made by the weighted sum of the immaculate photospheric spectrum $F_{\mathrm{ph}}(\lambda)$ and the spot spectrum $F_{\mathrm{sp}}(\lambda)$, that, neglecting the limb darkening, can be expressed as

$F_{\text {comp }}=A_{\text {rel }} F_{\mathrm{sp}}(\lambda)+\left(1-A_{\text {rel }}\right) F_{\mathrm{ph}}(\lambda)$.

For such a composite spectrum the line-depth ratio $r_{\text {comp }}$ can be expressed as follows

$$
\begin{aligned}
r_{\mathrm{comp}}= & \frac{A_{\mathrm{rel}} \frac{F_{\mathrm{sp}}^{\mathrm{c}}}{F_{\mathrm{ph}}^{\mathrm{c}}} d_{1}^{\mathrm{sp}}+\left(1-A_{\mathrm{rel}}\right) d_{1}^{\mathrm{ph}}}{A_{\mathrm{rel}} \frac{F_{\mathrm{sp}}^{\mathrm{c}}}{F_{\mathrm{ph}}^{\mathrm{c}}} d_{2}^{\mathrm{sp}}+\left(1-A_{\mathrm{rel}}\right) d_{2}^{\mathrm{ph}}} \\
= & \frac{A_{\mathrm{rel}} \frac{F_{\mathrm{sp}}^{\mathrm{c}}}{F_{\mathrm{ph}}^{\mathrm{c}}} r^{\mathrm{sp}}+\left(1-A_{\mathrm{rel}}\right) r^{\mathrm{ph}} \beta}{A_{\mathrm{rel}} \frac{F_{\mathrm{sp}}^{\mathrm{c}}}{F_{\mathrm{ph}}^{\mathrm{c}}}+\left(1-A_{\mathrm{rel}}\right) \beta},
\end{aligned}
$$

where $\beta=\frac{d_{2}^{\mathrm{ph}}}{d_{2}^{\mathrm{p}}}$. Since $d_{2}$ is almost insensitive to temperature, $\beta$ is very close to unity, as we found in all line pairs used by us. Given the almost linear dependence of line-depth ratios with respect to $T_{\text {eff }}$ in all the temperature range between photospheric $(\approx 5000 \mathrm{~K})$ and presumed spot $(\approx 3700 \mathrm{~K})$ temperature (see Paper I), we can replace $r$ with $T$ in Eq. (A.3) which becomes

$$
T_{\text {comp }} \simeq \frac{A_{\text {rel }} \frac{F_{\mathrm{sp}}^{\mathrm{c}}}{F_{\mathrm{ph}}^{\mathrm{c}}} T_{\mathrm{sp}}+\left(1-A_{\mathrm{rel}}\right) T_{\mathrm{ph}}}{A_{\mathrm{rel}} \frac{F_{\mathrm{sp}}^{\mathrm{c}}}{F_{\mathrm{ph}}^{\mathrm{c}}}+\left(1-A_{\mathrm{rel}}\right)}
$$

and can be written, at each orbital phase, as

$$
T_{\mathrm{m}}(\phi)=\frac{\gamma(\phi) T_{\mathrm{sp}}+T_{\mathrm{ph}}}{\gamma(\phi)+1}
$$

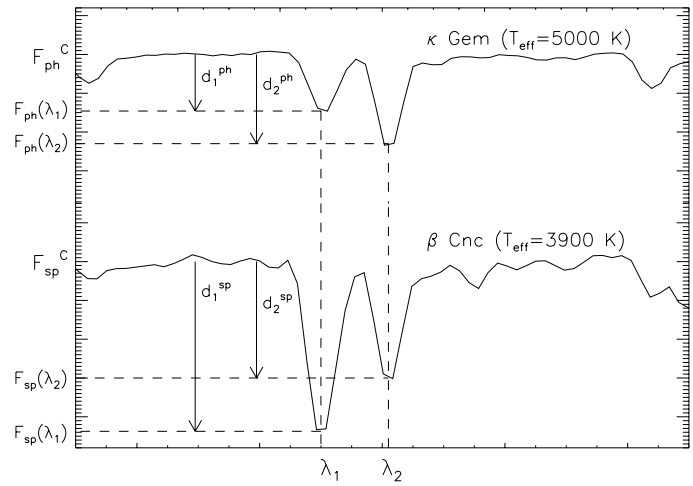

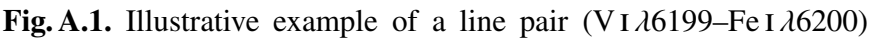
in spectra of standard stars with temperatures typical of the photosphere of an active early-K star (upper plot) and of a starspot (lower plot). The line-depth of both lines and the continuum fluxes, used in Eq. (A.1)-(A.4), are also indicated.

where $\gamma(\phi)=\frac{A_{\mathrm{rel}}(\phi)}{1-A_{\mathrm{rel}}(\phi)} \frac{F_{\mathrm{sp}}^{\mathrm{c}}}{F_{\mathrm{ph}}^{\mathrm{c}}}$ is the continuum contrast parameter of the spot against the quiet photosphere at the line pair wavelength. It is easy to note the interplay of fractional area of the starspots and the flux ratio in affecting the "observed" average temperature.

The relative luminosity, given as the ratio between the luminosity at the rotational phase $\phi$ and maximum (unspotted) luminosity, is

$L_{\mathrm{rel}}(\phi)=\frac{\int F(\phi) \mathrm{d} A}{\int F_{\text {unsp }} \mathrm{d} A} \simeq\left(1-A_{\mathrm{rel}}(\phi)\right)+A_{\mathrm{rel}}(\phi) \frac{F_{\mathrm{sp}}^{\mathrm{c}}}{F_{\mathrm{ph}}^{\mathrm{c}}}$,

where $L_{\mathrm{rel}}(\phi)=10^{-0.4 \Delta V(\phi)}$ and $\Delta V(\phi)$ is the differential light curve, taken with respect to the value at maximum brightness. In this case, $L_{\mathrm{rel}}(\phi)$ depends on the presumed unspotted magnitude, on the spot area and it is also a function of $T_{\mathrm{sp}} / T_{\mathrm{ph}}$ through $F_{\mathrm{sp}} / F_{\mathrm{ph}}$, but with a very different behavior with respect to the average temperature. So the interplay of fractional spot area and flux ratio is still present but with a different impact on the light curve.

We would like to stress that the continuum flux ratio depends on the observing wavelength, so that the relative contribution of a cool spotted region to the observed flux is higher at redder wavelengths and, as a consequence, would produce a stronger temperature variation. Vice versa, the same flux ratio enhancement would make the amplitude of the light curve smaller at redder wavelengths.

We have verified whether the simultaneous solution of temperature and light curves is able to reproduce the individual LDR curves. For this purpose we built up a set of "composite" spectra, at different rotational phases, simulating the HK Lac starspot distribution (Table 4).

The spectra of $\epsilon$ Cyg (K0 III, $\left.T_{\text {eff }}=4795 \mathrm{~K}\right)$ and $\beta \mathrm{Cnc}$ (K4 III, $T_{\text {eff }}=3965 \mathrm{~K}$ ) have been used to reproduce the "unspotted" photosphere and the spots of HK Lac, respectively.

Four observed LDR curves are displayed in Fig. A.2 as filled circles. The LDR curves from synthetic spectra are displayed with dashed lines. The full lines represent the same curves after the correction for the offset between the observed and synthetic curves which is mainly produced by the lower 
A. Frasca et al.: Measuring starspot temperature from line-depth ratio. II., Online Material p 3

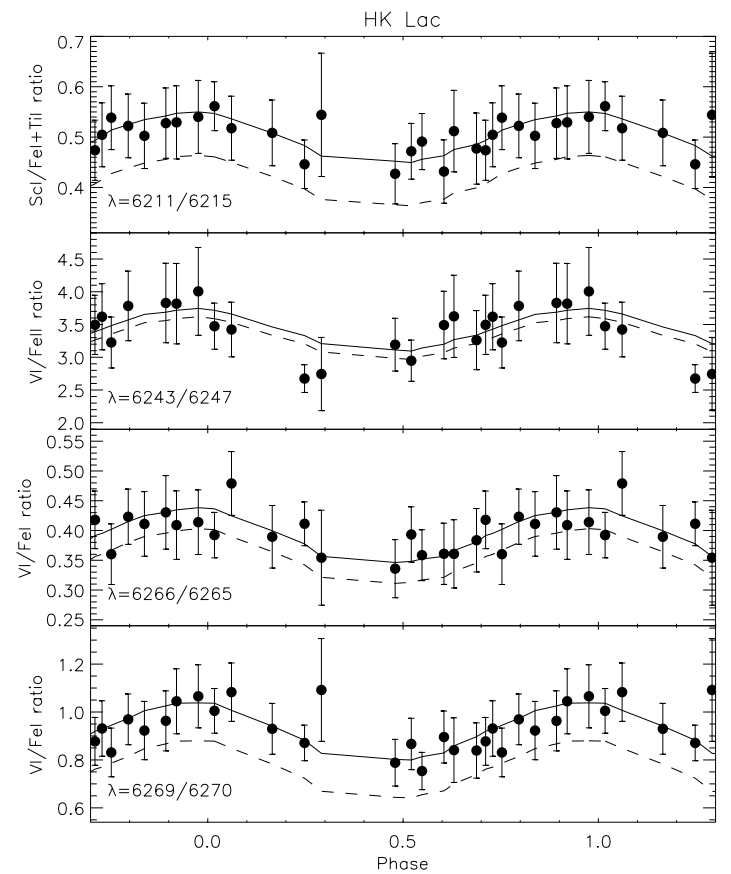

Fig. A.2. Observed (dots) and synthesized LDRs (dashed lines) for HK Lac. The LDR curves corrected for the small offsets are displayed with full lines.

gravity of $\epsilon$ Cyg with respect to HK Lac. All LDRs shown in Fig. A.2 display also a non-negligible sensitivity to gravity (see Paper I) that is in agreement with the observed shifts.

Apart from the aforementioned offset, the variation amplitudes reproduce very well the observed ones. This result confirms that the application of a spot model to a single $T_{\text {eff }}$ curve is a viable method for starspot temperature measurements. 